\title{
Standardization and Detailed Characterization of the Syngeneic Fischer/F98 Glioma Model
}

\author{
David Mathieu, Roger Lecomte, Ana Maria Tsanaclis, Annie Larouche, \\ David Fortin
}

\begin{abstract}
Introduction: Adequate animal glioma models are mandatory for the pursuit of preclinical research in neuro-oncology. Many implantation models have been described, but none perfectly emulate human malignant gliomas. This work reports our experience in standardizing, optimizing and characterizing the Fischer/F98 glioma model on the clinical, pathological, radiological and metabolic aspects. Materials and methods: F98 cells were implanted in 70 Fischer rats, varying the quantity of cells and volume of implantation solution, and using a micro-infusion pump to minimize implantation trauma, after adequate coordinates were established. Pathological analysis consisted in hematoxylin and eosin (H\&E) staining and immunohistochemistry for GFAP, vimentin, albumin, TGF-b1, TGFb2, CD3 and CD45. Twelve animals were used for MR imaging at 5, 10, 15 and 20 days. Corresponding MR images were compared with pathological slides. Two animals underwent ${ }^{18} \mathrm{~F}-\mathrm{FDG}$ and ${ }^{11} \mathrm{C}$-acetate PET studies for metabolic characterization of the tumors. Results: Implantation with $1 \times 10^{4}$ cells produced a median survival of 26 days and a tumor take of $100 \%$. Large infiltrative neoplasms with a necrotic core were seen on H\&E. Numerous mitosis, peritumoral infiltrative behavior, and neovascular proliferation were also obvious. GFAP and vimentin staining was positive inside the tumor cells. Albumin staining was observed in the extracellular space around the tumors. CD3 staining was negligible. The MR images correlated the pathologic findings. ${ }^{18} \mathrm{~F}$-FDG uptake was strong in the tumors. Conclusion: The standardized model described in this study behaves in a predictable and reproducible fashion, and could be considered for future pre-clinical studies. It adequately mimics the behavior of human malignant astrocytomas.
\end{abstract}

RÉSUMÉ: Standardisation et caractérisation détaillée du modèle de gliome syngénique Fischer/F98. Contexte : Nous avons besoin de modèles animaux adéquats pour la recherche préclinique sur le gliome en neuro-oncologie. Plusieurs modèles d'implantation ont été décrits, mais aucun ne correspond parfaitement aux gliomes malins chez l'humain. Nous rapportons notre expérience de standardisation, d'optimisation et de caractérisation du modèle de gliome Fisher/F98 du point de vue clinique, anatomopathologique, radiologique et métabolique. Matériels et méthodes : Des cellules F98 ont été implantées chez 70 rats Fisher, tout en variant la quantité de cellules et le volume de solution d'implantation au moyen d'une pompe à microinfusion afin de minimiser le traumatisme dû à l'implantation, après avoir établi des paramètres adéquats. En anatomopathologie, nous avons utilisé la coloration H\&E et l'immunohistochimie pour la GFAP, la vimentine, l'albumine, le TGF-b1, le TGF-b2, le CD3 et le CD45. Douze animaux ont subi une IRM aux jours 5, 10, 15 et 20. Les images ont été comparées aux lame histopathologiques correspondantes. On a procédé à des études au moyen du PET scan avec les traceurs 18F-FDG et 11C-acétate afin d'étudier le métabolisme des tumeurs chez deux animaux. Résultats : Les animaux chez qui on a implanté 1 x104 cellules ont eu une survie médiane de 26 jours et une prise d'implant de 100\%. À la coloration H\&E, on a observé de larges néoplasmes infiltrants avec un centre nécrotique, ainsi que de nombreuses mitoses, un comportement infiltrant péritumoral et une prolifération néovasculaire. La coloration pour la GFAP et la vimentine étaient positives dans les cellules tumorales. La coloration pour l'albumine était positive dans les espaces extracellulaires autour des tumeurs. La coloration CD3 était négligeable. L'IRM était corrélée aux observations anatomopathologiques. La captation du 18F-FDG dans les tumeurs était importante. Conclusion : Le modèle standardisé décrit dans cette étude se comporte de façon prévisible et reproductible et pourrait être utilisé à l'avenir dans les études précliniques. Il simule adéquatement le comportement des astrocytomes malins de l'humain.

Can. J. Neurol. Sci. 2007; 34: 296-306

Malignant gliomas are aggressive brain tumors with a particularly poor prognosis. The standard treatment, consisting of maximal resective surgery followed by radiation and chemotherapy at recurrence, offers at best palliative control. Survival generally ranges from a few months to about 15 months for glioblastoma multiforme, the most aggressive of these lesions. ${ }^{1}$ Many therapeutic strategies are currently under
From the Department of Surgery, Division of Neurosurgery and Neuro-oncology (DM, DF, AL), Department of Pathology (AMT), Department of Radiobiology (RL), Centre Hospitalier Universitaire de Sherbrooke, Sherbrooke University, Sherbrooke, Quebec, Canada.

ReCEIVED OCTOBER 2, 2006. ACCEPTED IN FINAL FORM MAY 5, 2007. Reprint requests to: David Fortin, Department of Surgery, Division of Neurosurgery and Neuro-oncology, CHUS, Sherbrooke University, Sherbrooke, Quebec, J1H 5N4, Canada. 
investigation, both in preclinical and clinical settings. For in vivo preclinical experimentation, the use of a representative animal glioma model is mandatory. Many models have been developed for this purpose using different approaches. Implantation models, derived from cultured cell lines that are implanted in the brain of the target animal, are the most widely used. ${ }^{2}$ However, a great number of these models tend to neglect some essential characteristics that can limit the way they emulate the behavior of their human counterparts. In order to optimize these models, some determinants should be carefully sought. ${ }^{2-5}$

The implantation technique should be relatively non traumatic, with minimal brain parenchymal damage and should have a low morbidity and no mortality for the host. The procedure should yield a tumor take rate close to $100 \%$. The developing tumors should present a growth rate that is relatively constant and reproducible among the different animals. As most brain tumors develop in relatively immunocompetent people, and given the fact that immune modulation is a potential therapeutic approach in the treatment of brain tumors, the neoplastic cells should be implanted in immunocompetent hosts rather than nude animals. Thus, in order to avoid any undesired immune reaction producing inherent tumor rejection, an essential quality of any implantation model is syngeneicity between the tumor cell line and the host. ${ }^{2}$ In addition, the glioma model should be relatively resistant to the available treatment strategies, as are human malignant gliomas. Finally, to facilitate in vivo imaging with noninvasive modalities such as magnetic resonance (MR) and positron emission tomography (PET), the implantation should be suitably located to avoid adjacent structures that could interfere with the tumor model in the images.

Very few of the current animal models described in the literature meet all these criteria. Many authors using a given rat strain use different implantation coordinates and techniques. ${ }^{6}$ Reported tumor take is highly variable from study to study, ranging from $50 \%$ to $100 \% .^{7-9}$ A considerable variation in tumor volumes can also be observed within a group of implanted animals using the same technique. A recently published study reported tumor volume after a constant observation period ranging from less than $10 \mathrm{~mm}^{3}$ to more than $80 \mathrm{~mm}^{3}$, despite standardization in the implantation technique..$^{10}$ Interestingly, the most widely used model, the C6/Wistar allogeneic model, has been shown to generate important humoral and cellular immune responses after tumor implantation, leading to spontaneous tumor regression. ${ }^{6,11,12}$ Unfortunately, spectacular in vivo experimental results using these models have not translated so far in any real therapeutic breakthrough for malignant glioma patients.

In trying to define and characterize an optimal model, we elected to work with the Fischer/F98 syngeneic model. The F98 cell line is an anaplastic glioma with a minor sarcomatous component that was originally produced by a single N-ethyl-Nnitrosourea injection to a 20 -week pregnant Fischer rat. ${ }^{2}$ The offspring developed brain tumors that were harvested and maintained in culture. These cells have depicted low immunogenicity when implanted in their syngeneic host, and consequently to their implantation, animals develop infiltrative tumors that are very resistant to conventional treatment. ${ }^{2}$ This thus translates the clinical situation with human malignant gliomas.
In the present work, we have extensively characterized the Fischer/F98 model clinically and pathologically, emphasizing the standardization and precision of the implantation process. We have also detailed radiological and metabolic assessment of the model.

\section{Materials ANd Methods}

\section{Cell Culture}

The F98 cell line was obtained from ATCC, and was grown in monolayer using a solution of Dulbecco's modified Eagle medium (DMEM) supplemented with $10 \%$ fetal bovine serum (FBS) and a mix of penicillin-streptomycin. Cells were incubated at $37^{\circ} \mathrm{C}$ in a humidified environment with $5 \% \mathrm{CO}_{2}$ and propagated upon confluence, every three days.

The implantation solution was prepared by trypsinization of the cell culture followed by resuspension in a DMEM solution free of FBS. Briefly, after washing twice with PBS, the adherent cell lines were detached from the Petri dishes by the addition of $1 \mathrm{ml}$ of $0.05 \%$ trypsin- $0.02 \%$ EDTA. The trypsin was then inactivated using $9 \mathrm{ml}$ of DMEM without FBS. The dilutions and volumes of the suspension solution were varied according to the scheme described in the implantation parameters adjustment section. A trypan blue exclusion test was performed to assess cell viability before implantation.

\section{Animals and study groups}

Adult male Fischer rats weighting 225 to 250 grams were acquired from Charles-River laboratories (Montreal, Quebec). The study group consisted of 70 animals, which were kept in our facilities. Animal care and experimentation were conducted in accordance with the recommendations of the Canadian Council on Animal Care and with the approval of the institutional animal experiment review board. Three study groups were designed: Group $1(n=56)$, the largest group, which served to study implantation techniques and parameters, clinical progression and animals survival; Group $2(\mathrm{n}=12)$, which was used to study MR and pathological correlation in the model after having defined a set of optimal implantation parameters; and Group $3(n=2)$, in which the metabolic studies were performed using PET imaging. Group 1 was further divided in subgroups (a,b,c,d,e - see implantation parameters adjustment section).

\section{Implantation technique}

Anesthesia was induced by inhalation of a mixture of oxygen with $5 \%$ halothane, followed by an intra-peritoneal injection of ketamine $(87 \mathrm{mg} / \mathrm{kg})$ and xylazine $(13 \mathrm{mg} / \mathrm{kg})$ for maintenance. Animals were then mounted on a stereotactic frame. Special care was used in the placement of the head in the frame to avoid sagittal angulation, so that the head was parrallel to the frame stand (Figure 1). A midline scalp incision was performed, followed by identification and exposure of the bregma. Using a 16-gauge needle, a burr hole was placed on the right frontal bone. A 25-microliter SGF syringe with a 27-gauge needle secured to the frame was used to infuse the cellular solution over a period of five minutes. The needle was then slowly withdrawn. Bone wax was applied to close the burr hole and the scalp was closed with a continuous one-layer resorbable suture. 


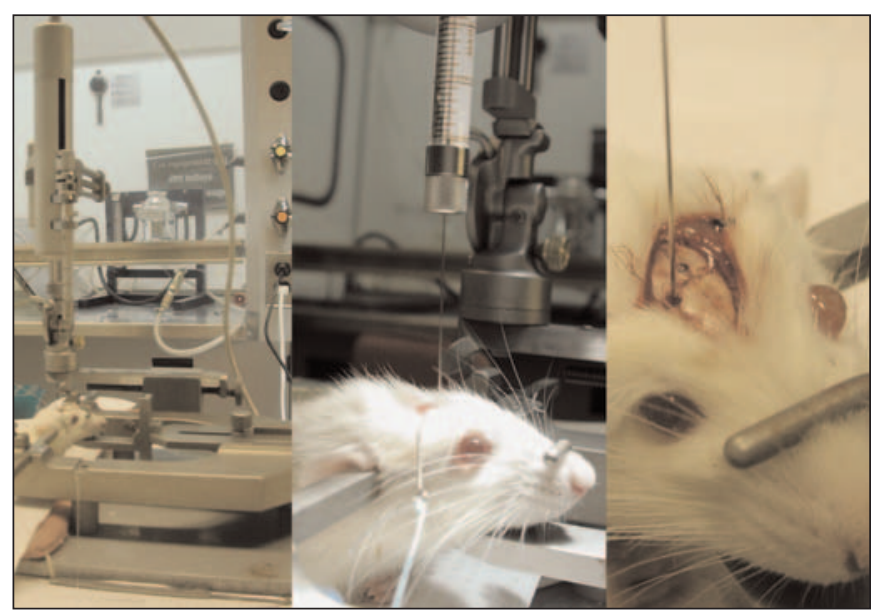

Figure 1: Final setup for the implantation. a) The micro-infusion pump is mounted on the stereotactic frame to allow a continuous and slow infusion rate. b) Once again care is used in the placement of the animal's head in the frame as even a slight sagittal mis-angulation will significantly affect the implantation coordinates. c) The burr hole was accomplished using a 16 gauge needle after coordinates localization using the stereotactic frame. A 27 gauge needle is used to deliver the implantation solution.

\section{Implantation parameters adjustment (Group 1)}

Serial experiments were performed to assess the implantation coordinates and cellular suspension characteristics that would lead to a valid and reproducible model with an optimal timeframe from implantation to death.

The first step consisted in securing adequate implantation coordinates. The pursued goal was to implant in the frontal lobe, away from the ventricles, and at a significant distance from the Harderian glands, exocrine glands located in the medial aspect of the orbit. These glands are highly metabolic, and uptake of the $\left[{ }^{18} \mathrm{~F}\right]$ fluorodeoxyglucose used in pet scanning the animals could potentially obscure the tumor uptake. ${ }^{13}$ Using the bregma as a reference landmark, anterior (0 to $3 \mathrm{~mm}$ ) and lateral (1 to $4 \mathrm{~mm}$ ) coordinates were tested by intracerebral infusion of an Evans blue solution using a stereotactic frame. Depth of implantation was also assessed, varying from a 5 to $7 \mathrm{~mm}$ depth to the external table of the skull. Once the Evans blue solution had been infused, the animals were euthanized, brain specimens were harvested, cut in the coronal plane and assessed for Evans blue localization (Figure 2).

The cellular concentration and volume of the implantation solution were also tested serially. Implantation procedures were initially conducted with volumes of 10 micro liters containing $5 \times 10^{5}$ (subgroup a, $\mathrm{n}=7$ ) or $2 \times 10^{5}$ (subgroup b, n=11) tumor cells. Volumes of 5 microliters with $1 \times 10^{5}$ (subgroup c, $n=13$ ) or $1 \times 10^{4}$ (subgroup $\mathrm{d}, \mathrm{n}=15$ ) cells, and one microliter containing $1 \times 10^{3}$ cells (subgroup e, $n=10$ ) were then evaluated.

The final parameter tested was the method of infusion. Initially, when testing the 10 microliters suspensions, manual injection of the solution was accomplished. In an effort to standardize infusion time, ensure a constant rate, and decrease the implantation traumatism, a micro-infusion pump (UltraMicroPump, World Precision Instruments Inc.) was acquired and was used to deliver the implantation solution for all the subsequent experiments (Figure 1).

\section{Final implantation parameters}

After analysis of the Evans blue study, the optimal implantation coordinates chosen were as follow: $1 \mathrm{~mm}$ anterior and $3 \mathrm{~mm}$ lateral to the right of the bregma, with solution infusion at a depth of $6 \mathrm{~mm}$ from the outer table of the skull. Based on the survival interval obtained after implantation and on the pathological examination of the specimens, we elected to work with a final solution concentration of $1 \times 10^{4}$ cells diluted in a volume of 5 microliters. The solution was delivered at a

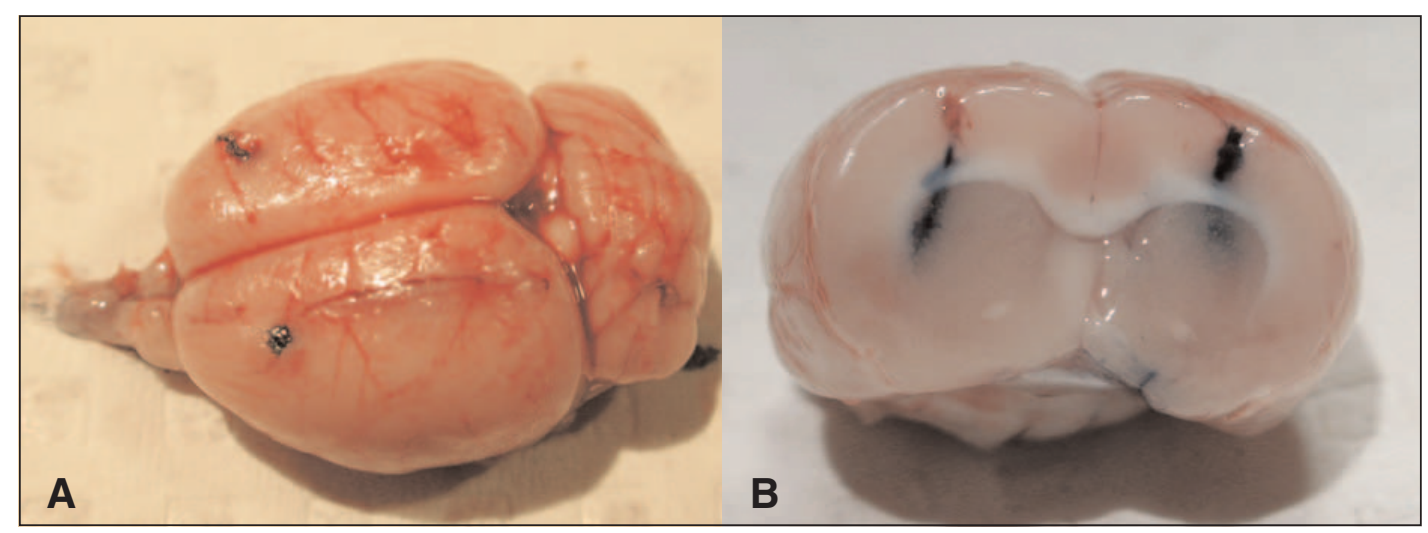

Figure 2: a) Brain specimen harvested after implantation coordinates testing using Evans blue as a marker. The entry points are noticeable. For standardization, the stereotactic frame was used in all procedures, and great care was used to place the head of the animal in the frame in an horizontal fashion, minimizing any sagittal angulation, so that implantation coordinates would be reproducible. b) Coronal cut of the brain showing the depth of the projected implantation coordinates and the implantation tracts. A depth of $6 \mathrm{~mm}$ was used on the left side, whereas a $4 \mathrm{~mm}$ depth was used on the right side. 
constant rate of one microliter per minute, over 5 minutes, using a micro-infusion pump, after which the needle was slowly withdrawn over one minute, to minimize backflow of the suspension solution.

\section{Post-procedure monitoring and euthanasia technique}

Animals were allowed to recover from the procedure, and were given food and water ad libitum afterward. They were assessed clinically bi-daily for the apparition of signs of raised intracranial pressure (lethargy, vomiting, and cachexia) or focal neurological signs (hemiparesis, ataxia). The subjects were weighted weekly and prior to euthanasia. The endpoint of the study was profound lethargy, and animals were euthanized once that state was installed. Euthanasia was carried by intracardiac perfusion of a $300 \mathrm{ml}$ solution of glutaraldehyde under general anesthesia, after having severed the splenic vein. The brain was immediately retrieved following the intracardiac perfusion.

\section{Specimen processing}

Upon retrieval, brain specimens were fixed in a formalin solution for 48 hours, cut in the coronal plane in $1 \mathrm{~mm}$-width slices using a dedicated brain matrix and embedded in paraffin. The blocks were sectioned at 3 micrometers intervals and the resulting slides were stained with hematoxylin and eosin (H\&E). After deparaffinization and dehydration, a microwave antigen retrieval process was performed. Slides were placed in 0.1 $\mathrm{mmol} / \mathrm{l}$ citrate buffer in a microwaveable pressure cooker and boiled in a 700-W microwave oven for 30 minutes. Sections were incubated with primary antibody. Biotinylated speciesspecific secondary antibodies were applied followed by an avidin-biotin amplification and peroxides development. Monoclonal antibody labeling was obtained for GFAP (BD bioscience, San Jose, California, dilution 1/10), vimentin (BD bioscience, San Jose, California dilution 1/100), TGF-b1 (Santa Cruz biotechnology, Santa Cruz, California, dilution 1/100), TGF-b2 (Santa Cruz biotechnology, Santa Cruz, California, dilution 1/80), albumin, CD3 (BD bioscience, San Jose, California dilution 1/175) and CD45 (BD bioscience, San Jose, California dilution 1/800).

\section{Pathologic analysis}

Slides were scanned at low-power magnification to identify the tumors, which were then examined at higher magnification. Tumor morphology and characteristics were assessed on H\&E. The number of mitotic figures per high-power field (HPF, 40X magnification) was noted for proliferation assessment. Immunohistochemistry labeling was assessed qualitatively for GFAP, vimentin, albumin, and TGF-b, and quantitatively for CD3 and CD45 (number of labeling cells per HPF).

\section{Radiological assessment (Group 2)}

Magnetic resonance imaging of the model was evaluated using a clinical 1.5T magnet (Siemens 1.5T magnetom Sonata with Syngo 2002B software). To determine the optimal imaging parameters, a series of preliminary images were performed on a non implanted test animal subject. Twelve rats were then randomly assigned to one of four groups, who were to be imaged at 5, 10, 15 and 20 days following tumor implantation.
Procedures were done under ketamin/xylazine anesthesia, as previously described. Animals were positioned prone in the magnet, with the antenna (Siemens small loop flex coil antenna) placed directly on the head. Coronal T2-weighted images and contrast-enhanced T1-weighted images were obtained. Following the imaging procedure, the animals were immediately sacrificed, as described in the previous section. Brains were harvested and processed for $\mathrm{H} \& \mathrm{E}$. To validate the MRI images, contrast-enhanced T1 images were compared with the corresponding $\mathrm{H} \& \mathrm{E}$ slides. The volume and largest crosssectional area on contrast imaging were assessed using the image-processing software Sigma Scan Pro 5 (Hallogram Publishing, Aurora, USA).

\section{Metabolic assessment (Group 3)}

Metabolic evaluation of the model was carried out by ${ }^{18} \mathrm{~F}$ FDG and 11C-acetate positron emission tomography (PET) scanning. The Sherbrooke small animal PET scanner (based on avalanche photodiode detectors) was used to acquire the images over the whole brain. ${ }^{14,15}$ With proper sampling motion, this scanner achieves $2.1 \mathrm{~mm}$ FWHM transaxial and 14 microliters volumetric resolution. ${ }^{16}$ Two animals underwent this imaging modality 14 days after the implantation. Scans were carried with the animals under general anesthesia as previously described. The animals were placed supine on the bed of the camera and kept warm with a heating pad. Prior to imaging, the camera was calibrated with a phantom filled with water containing $0.98 \mathrm{MBq}$ $(700 \mathrm{uCi})$ of ${ }^{18} \mathrm{~F}-\mathrm{FDG}$ in a volume of $22 \mathrm{ml}$. Dynamic imaging was initiated $1 \mathrm{~min}$ prior to injection of the isotopes, and continued for $1830 \mathrm{sec}$, followed by 3 (1289 sec) volumetric acquisitions. The images were reconstructed using 25 iterations of the maximum likelihood expectation maximization algorithm implementing position-dependent detector response. ${ }^{17}$ Isotope uptake was analyzed on the reconstructed images using the Sherbrooke LabTEP software (Benard F, Rousseau E, Sherbrooke LABTEP software, v. 1.25 release 3, November 2003). Briefly, a region of interest (ROI) was drawn over the tumor and from an equivalent size ROI traced over an area of healthy brain tissue. Results were expressed as the tumor to healthy brain tissue uptake ratio.

\section{Results}

\section{Clinical data (Group 1)}

Fifty-six rats underwent F98-glioma implantation and were subsequently clinically followed. Animals developed symptoms of a brain mass following a time-frame that varied according to the number of tumor cells implanted. Symptoms consisted of progressive lethargy with variable degree of hemiplegia contralateral to the implantation side. In the later phases, ataxic irregular pattern of breathing could be observed. A significant majority of subjects lost weight over the course of their evolution, with a mean of $5.2 \%$ body weight loss. Two animals in subgroup e ( $1 \times 10^{3}$ cells) never developed symptoms and were sacrificed after 50 days had elapsed. These subjects were excluded from survival analysis. As expected, survival of the animals was inversely related to the number of cells implanted (Table 1). Animals in subgroup $\mathrm{d}\left(1 \times 10^{4}\right.$ cells $)$ had a median survival of 26 days. An animal belonging to this group was 
Table 1: Survival time according to the number of implanted tumor cells

\begin{tabular}{c|c|c|c|c}
\hline \hline $\begin{array}{c}\text { Number of } \\
\text { implanted } \\
\text { cells }\end{array}$ & $\begin{array}{c}\text { Number of } \\
\text { animals }\end{array}$ & $\begin{array}{c}\text { Median } \\
\text { survival } \\
\text { (days) }\end{array}$ & $\begin{array}{c}\text { Mean } \\
\text { survival } \\
\text { (days) }\end{array}$ & $\begin{array}{c}\text { Standard } \\
\text { deviation }\end{array}$ \\
\hline $5 \times 10^{5}$ & 7 & 16 & 12.4 & 5.0 \\
\hline $2 \times 10^{5}$ & 11 & 15 & 16.5 & 2.7 \\
\hline $1 \times 10^{5}$ & 13 & 18 & 18.1 & 2.8 \\
\hline $1 \times 10^{4}$ & $12^{1}$ & 26 & 25.3 & 4.6 \\
\hline $1 \times 10^{3}$ & $8^{2}$ & 36 & 35.0 & 4.7 \\
\hline
\end{tabular}

${ }^{13}$ animals excluded, ${ }^{2} 2$ animals excluded

excluded from survival analysis because of precocious death on the seventh post-implantation day. Pathological examination revealed a very small tumor growing in the implantation site, devoid of any mass effect. The death of this animal was deemed unrelated to the tumor or the procedure.

Implantation procedures were generally well tolerated. Two animals were found dead the day following the intervention. One of the specimens depicted a hemorrhage at the surgical site and along the implantation tract. The other was postulated to have died from an anesthetic complication as no pathologic finding was uncovered at autopsy. These two animals were also excluded from tumor take and survival analysis. Otherwise, no clinicallysignificant morbidity occurred after the procedure. Thus, total procedure-related morbidity, including animals from Groups 1, 2 and 3 , was $2.9 \%(2 / 70)$.

\section{$H \& E$ examination (Group 1, 2 and 3)}

The specimen examination revealed the presence of a significant tumor in 66 of a total of 68 eligible animals, thus producing a tumor take of $97.1 \%$. Tumors were large, with a central necrotic core and numerous mitotic figures, with a mean of 8 mitoses per HPF. Obvious distortion in the cerebral architecture was accompanied by important brain shift and mass effect (Figure 3). Vascular proliferative changes could be observed in most specimens. Perivascular neoplastic infiltration was obvious, as was the adjacent brain parenchymal infiltration by individual tumor cells at the edge of the lesion, in all samples (Figure 4). In the specimens implanted with solution volumes greater than 5 microliters, the tumor often extended along the implantation tract all the way to the surface of the brain, with leptomeningeal spread. This phenomenon was infrequently seen in the subgroups infused with volumes of 5 and 1 microliters, using the micro-infusion pump.

Tumor areas were evaluated using slides from Group 2, to assess tumor growth and variation between each Group. Tumor margin area measurements at pathology are reported in Table 2, against corresponding calculated areas at MRI. A gradual and consistent progression in areas was noted across the study period on the pathology specimens.

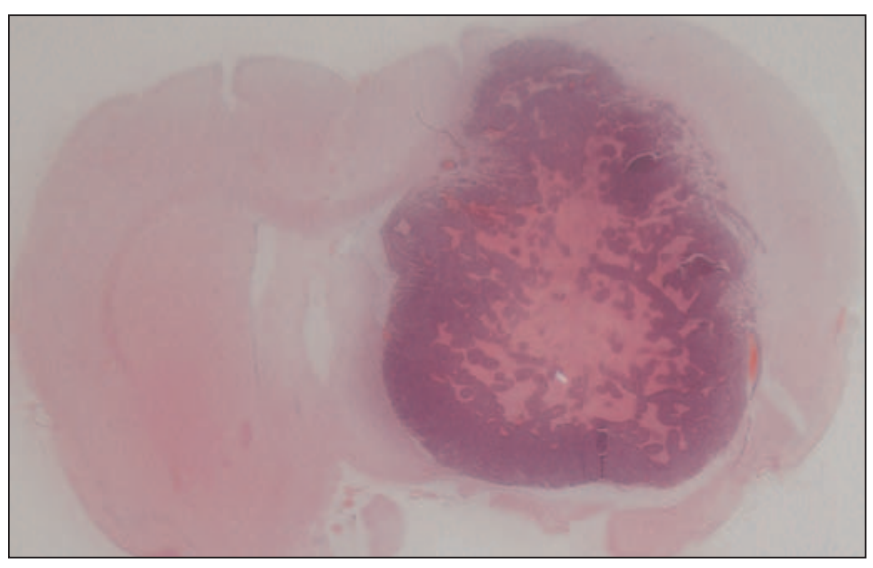

Figure 3: Coronal $H \& E$ slide of a representative specimen obtained using the defined optimal coordinates (Group 1d) and sacrificed at 25 days post-implantation, when the animal presented a lethargic condition. Notice the large right hemispheric tumor distorting the brain parenchyma and producing a significant midline shift. This tumor also depicts central necrosis, and peripheral brain infiltration.

Table 2: Tumor areas

\begin{tabular}{c|c|c}
\hline $\begin{array}{c}\text { Post- } \\
\text { implantation } \\
\text { interval (days) }\end{array}$ & $\begin{array}{c}\text { Pathologic sample } \\
\text { surfaces }\left(\mathrm{mm}^{2}\right)\end{array}$ & $\begin{array}{c}\text { Radiological image } \\
\text { surfaces }\left(\mathrm{mm}^{2}\right)\end{array}$ \\
\hline 5 & $0.29 \pm 0.2$ & $2.18 \pm 0.91$ \\
\hline 10 & $4.43 \pm 0.89$ & $8.25 \pm 0.91$ \\
\hline 15 & $8.3 \pm 0.22$ & $21.6 \pm 2.38$ \\
\hline 20 & $12.9 \pm 0.45$ & $23.17 \pm 2.56$ \\
\hline
\end{tabular}

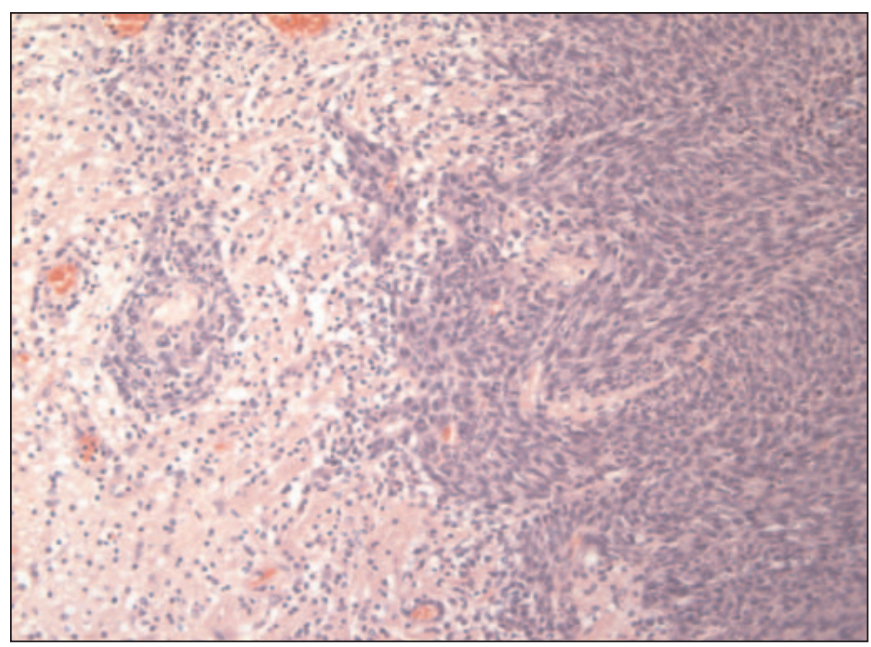

Figure 4: $H \& E$ slide $(40 X)$ of a representative specimen obtained using the defined optimal coordinates (Group 1d), focusing the field of view at the periphery of the tumor nodule. Infiltration of the adjacent brain parenchyma by neoplastic cells is obvious, as is a pattern of perivascular clustering. 


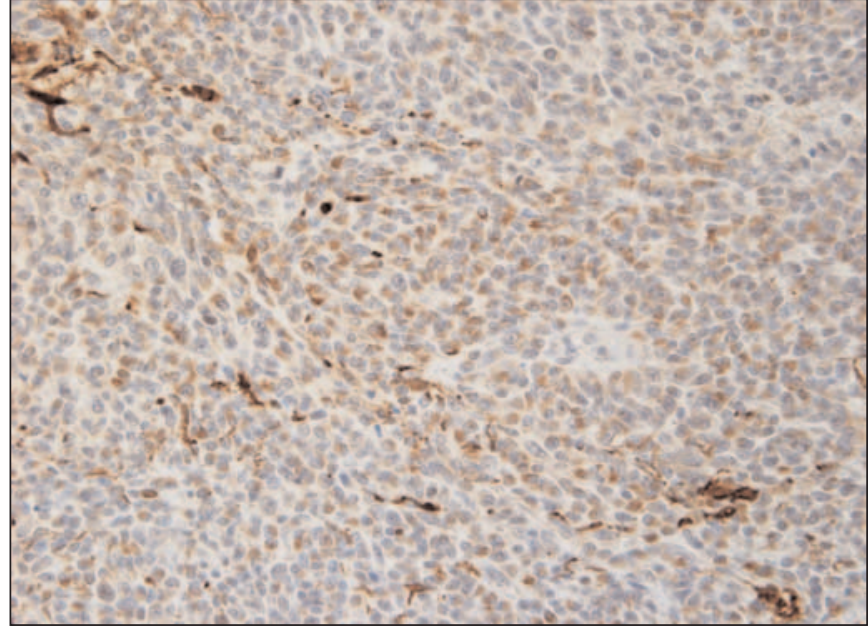

Figure 5: GFAP immunohistochemistry $(40 X)$ of a representative specimen. Widespread cytoplasmic staining for GFAP is obvious in numerous cells. The field of view is focused in the center of the tumor nodule.

\section{Immunohistochemistry (Group 1)}

Glial fibrillary acidic protein (GFAP) immunohistochemistry confirmed that a significant proportion of the neoplastic cells were of astrocytic origin (Figure 5). In addition, dense GFAP labeling was observed in the adjacent parenchyma surrounding the tumors, in reactive astrocytes. Vimentin expression was diffuse in the tumor cells (Figure 6). Profuse albumin immunostaining was observed in the extracellular space at the periphery of the tumors, an illustration of the pathologically increase in the blood-brain barrier permeability (Figure 7). When positive, labeling for CD3 cells was seen at the periphery of the tumor and around adjacent capillaries (Figure 8). A mean of 2.8 staining cells per HPF was observed. CD45 staining was

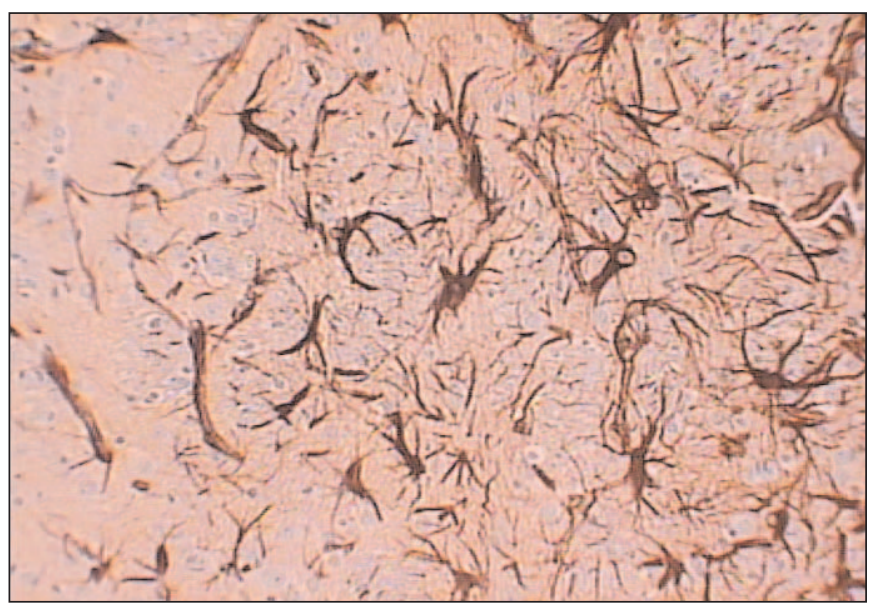

Figure 6: Vimentin immunohistochemistry $(40 \mathrm{X})$ of a representative specimen in the tumor. A widespread marking is present. The field of view is focused at the peripheral aspect of the tumor nodule.

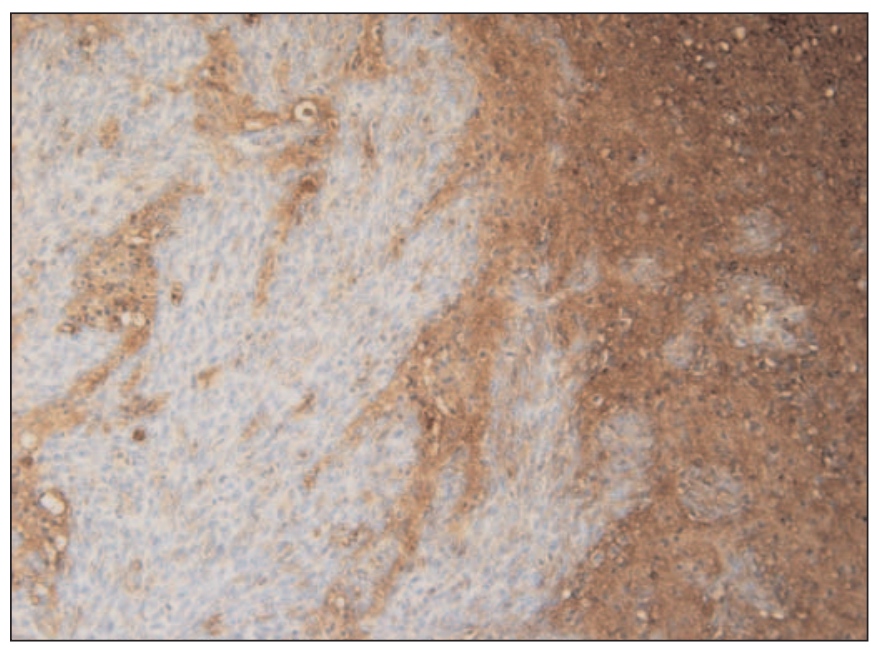

Figure 7: Albumin immunohistochemistry $(20 \mathrm{X})$ of a representative specimen sacrificed at 26 days post implantation. The field of view is centered at the periphery of the main tumor nodule to emphasize the abumin staining in the region of the brain around tumor.

extremely low, with only an occasional staining cell (not shown). Transforming growth factor beta 1 (TGF-b1) depicted perivascular staining in the tumor nodules, especially at the periphery (Figure 9). Occasional individual neoplastic cells depicted cytoplasmic staining. No significant TGF-b2 staining was observed.

\section{MRI evaluation (Group 2)}

Gadolinium-enhanced T1-weighted images depicted a clear brain-tumor interface in all the study specimens. Tumor progression was obvious and measurable over the study period. The average volume calculated for each time group was as follows: $6.15 \mathrm{~mm}^{3} \pm 2.74$ at 5 days, $15.9 \mathrm{~mm}^{3} \pm 7.0$ at 10 days,

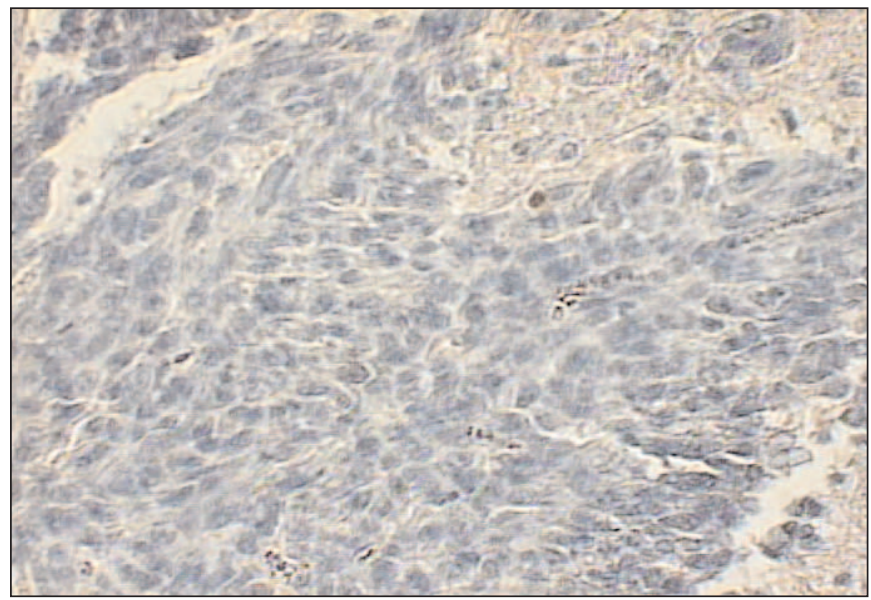

Figure 8: CD3 immunostaining $(40 X)$ at the peritumoral area, demonstrating scarce lymphocytic infiltration, with only a single cell staining in this typical sample. 


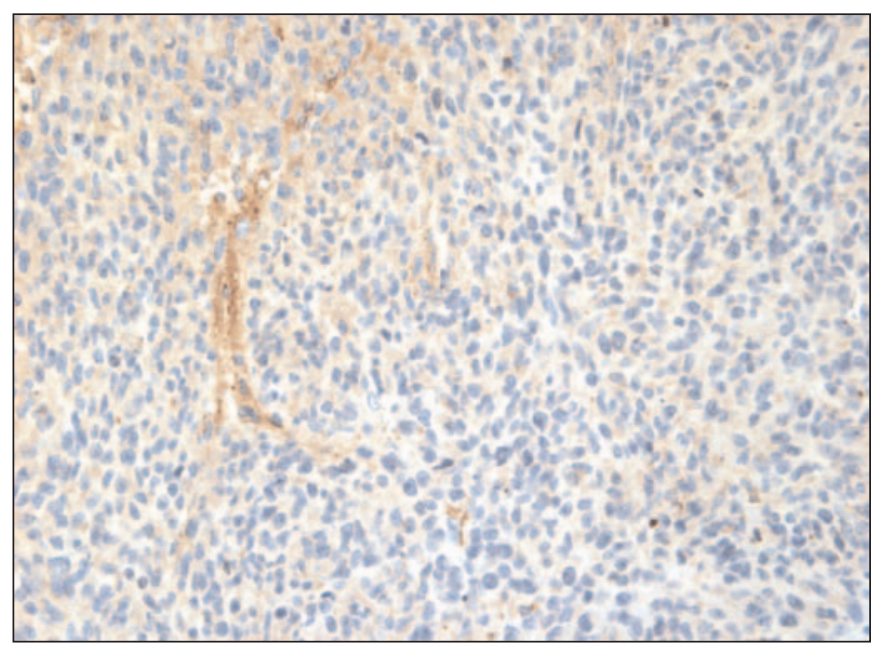

Figure 9: TGF-b1 immunostaining $(40 X)$ in the tumor nodule. Interestingly, although TGF-b1 stained diffusely in the extracellular matrix of the tumor sample (the protein is stored in the extracellular matrix once secreted), a predominant staining was identified around blood vessels, as can be seen in this sample. No staining was observed in the normal brain tissue (not shown).
$59.8 \mathrm{~mm}^{3} \pm 18.8$ at 15 days and $77.0 \mathrm{~mm}^{3} \pm 29.0$ at 20 days. Comparisons of the largest radiological and pathological crosssectional area demonstrated a parallel increase over time, although the MRI studies consistently overestimated tumor volumes (Figure 10). Moreover, and in a distinctive behavior to the pathology specimens, the growth pattern reached a threshold between day 15 to day 20 (Figure 11). T2-weighted images demonstrated significant peritumoral edema, with blurred tumor margins (Figure 12).

\section{Metabolic evaluation (Group 3)}

Positron emission tomography scanning studies demonstrated strong ${ }^{18} \mathrm{~F}$-FDG uptake by the tumors as compared to the adjacent brain parenchyma ( $\mathrm{F}$ igure 13 ). Harderian glands also demonstrated a significant uptake of the radiotracer, showing the importance of tumor implantation at a distance from those glands. For ${ }^{11} \mathrm{C}$-acetate no difference in uptake between tumor and surrounding brain was observed. Radiotracer uptake ratio was 1.12 for ${ }^{11} \mathrm{C}$-acetate, and 1.71 and 1.97 for ${ }^{18} \mathrm{~F}-\mathrm{FDG}$ at 30 and 50 minutes post-injection, respectively.

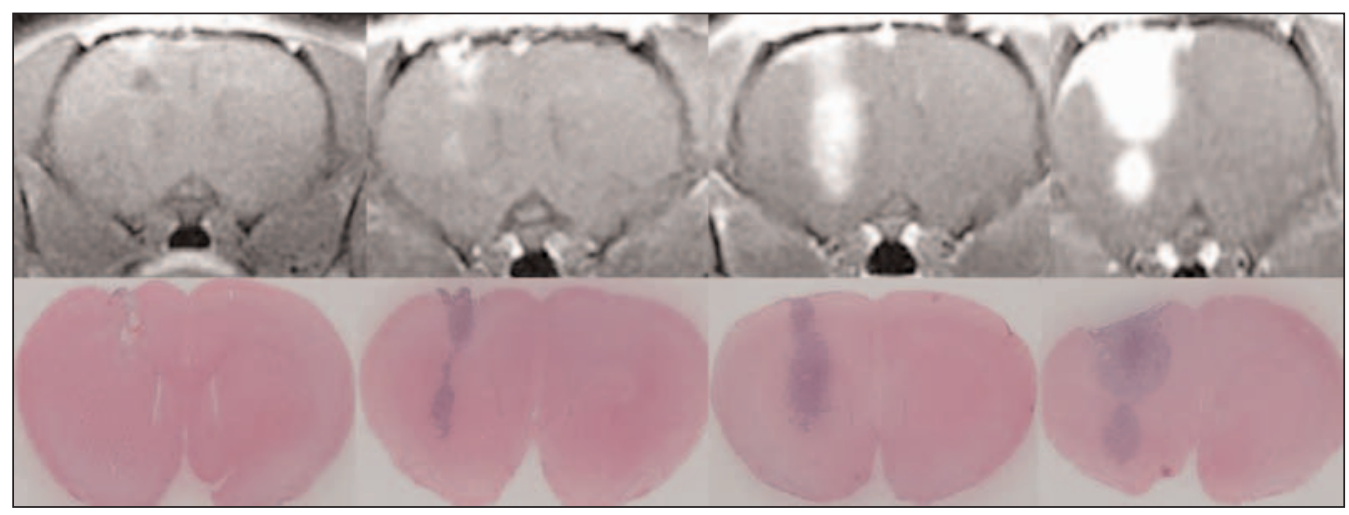

Figure 10: Composite picture displaying tumor progression in four animals of Group 2, at 5, 10, 15 and 20 days after implantation. Top row depict coronal gadolinium-enhanced T1 MRI at 5,10,15 and 20 days in four different animals, whereas bottom row match the corresponding pathology samples. The MRI adequately translates the general morphology of the tumors and progression over the observation period.

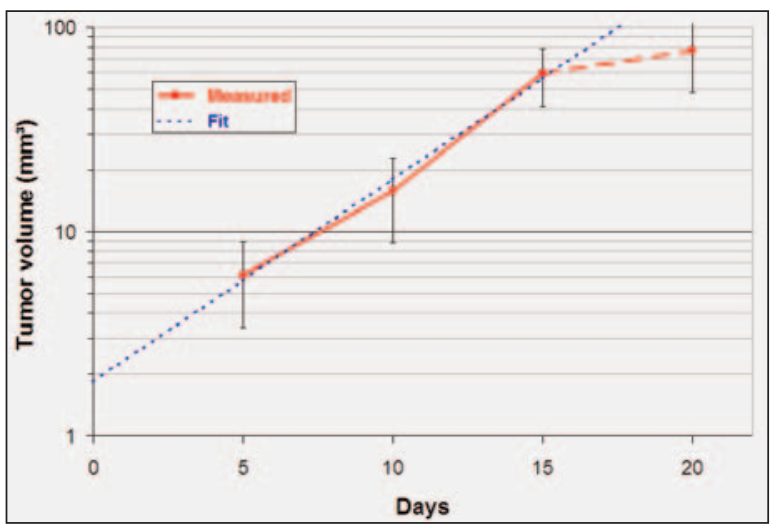

Figure 11: Graphic plotting tumor volumes obtained at 5, 10, 15 and 20 days against a logarithmic scale. A saturation is observed after 15 days, as the tumor growth slows.

\section{DISCUSSION}

Numerous rat glioma models have been described in the literature..$^{2,410-12,18-30}$ Although from a theoretical point of view, transgenic models might better emulate human tumors and present several advantages, ${ }^{3,4}$ in practice most researchers have used implantation models. This is largely attributable to their simplicity of use and relatively inexpensive cost compared to transgenic models, which require substantial resources to develop and handle.,4 Tumor implantation involves several critical steps which can adversely impact the model validity and reproducibility. These steps are too frequently occulted from discussion and dismissed as unimportant, with the consequence that the lack of standardization plagued a great number of these models. As a result, different implantation coordinates are used without validation, tumor takes are highly variable, and so are tumor volumes within a given study group. ${ }^{4,7-10}$ Given all these 


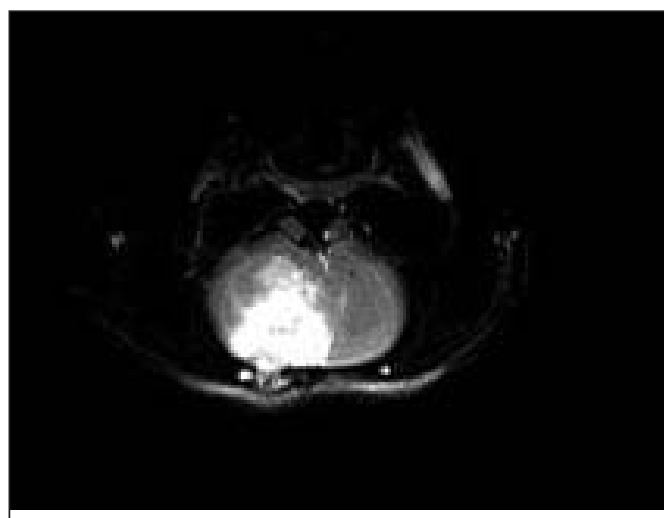

Figure 12: Coronal T2 restore sequence, with a field of view of $10.5 \mathrm{X}$ $10.5 \mathrm{~cm}$ and a matrix of $512 \times 384$, at 20 days after implantation in a Fischer rat. The hyperintense signal encompasses the tumor mass, as well as the surrounding edema.

variations, it can be difficult to draw reliable conclusions from therapeutic studies investigating tumor growth and/or tumor volumes. It is thus paramount to standardize all the steps involved in the elaboration of a glioma implantation model to minimize these procedural-related factors that can bias the model.

The selection of the appropriate cell line and host animal obviously represents the first step in the design of an implantation model. A large number of rodent malignant glial cell lines are commercially available, and models have been described using each of them. The C6 and 9L lines are the most widely used although both have obvious shortcomings when it comes to preclinical evaluation of therapeutic strategies. The C6 cell line is an infiltrative glioma which has no known syngeneic host and is usually implanted in the Wistar rat. ${ }^{2,4,6,19}$ Immune responses potentially leading to tumor rejection have been reported in these models. ${ }^{6,11,12}$ The $9 \mathrm{~L}$ cell line gives rise to sarcomatous tumors that often behave like metastatic lesions, with poor infiltration of surrounding brain parenchyma. ${ }^{2,31}$ Given these shortcomings, we believe these models are not ideally suited to study tumor growth and response to experimental treatments. Nonetheless, despite these limitations, these models which are well-characterized in the literature have important

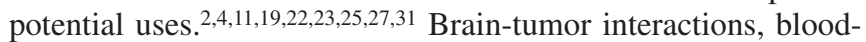
brain barrier assessment, metabolic studies, in vitro assays, among others are all valid applications of these models.

We chose to work with the F98 cell line for several reasons. It is an anaplastic glioma with a minor sarcomatous component. Its syngeneic host is well known to be the Fischer rat. ${ }^{2}$ A very weak immunogenicity characterizes this line, with reports of successful short-term implantations in cat brains. ${ }^{32}$ It is extremely stable in culture, with no morphological changes observed in over a hundred cell passages in our laboratory. Few treatment strategies have produced significant tumor regression when tested on this cell line after intracranial implantation in its

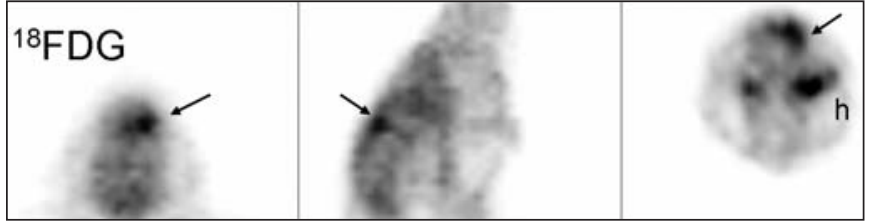

Figure 13: ${ }^{18} \mathrm{~F}-\mathrm{FDG}$ uptake is increased in the tumor (arrow) compared to adjacent brain, as well as in the Harderian glands $(h)$, as illustrated in this composite of coronal, sagittal and trans-axial acquisitions.

syngeneic host, and only a few investigators have reported survival improvement using this model in preclinical therapeutic trials. ${ }^{7,33-39}$ This aggressive behavior adequately parallels the clinical situation.

In designing the implantation procedure, the goals sought are the constant and reproducible production of tumors, growing in a predictable pattern, and a low morbidity. The mandatory use of a stereotactic frame for the procedure cannot be overemphasized. A freehand injection has already been reported to be inaccurate and to give rise to inconsistent tumors, ${ }^{2,5,25}$ but some investigators still use this approach for their models, as illustrated recently in a published work. ${ }^{31}$

In the present study, implantation coordinates were first assessed and validated using Evans blue (Figure 1). The area targeted was the region of the head of the caudate nucleus, in the frontal lobe far from the ventricle and somewhat at a distance from the Harderian glands. Once these coordinates were established, the volume and number of cells of the suspension solution were determined. The smallest infusion volume and the smallest number of cells were pursued, the rationale being that this would decrease the trauma associated to the implantation procedure, and thus the consequent inflammatory reaction. Moreover, we hypothesized that by delivering smaller volumes, we would increase the infiltrative behavior of the tumor cells by decreasing the artifactual cavity created by the implantation. For the same reason, the use of a micro-infusion pump to infuse the suspension solution was introduced. By allowing the slowest delivery possible, we hypothesized that this approach would decrease the risks of reflux of the solution along the implantation tract.

The implantation technique as described in this study depicted very low morbidity in our hands with only two animals suffering from periprocedural death in 70 implantations (2.9\%). It should be noted that no animal presented morbidity from the implantation procedure after the establishment of the optimal coordinates.

The tumor take was $100 \%$ when considering the animals implanted with the optimal coordinates (Group 1, subgroup d; Group 2 and 3). The overall tumor take was 66/68 when all the groups were considered. Group 1, subgroup e, consisting of ten animals in which $1 \mu \mathrm{l}$ of $1 \times 10^{3}$ cells were implanted, depicted no tumor growth in the first two animals implanted. We suspect that this occurred because of mechanical limitation in the infusion pump and inadequate syringe needle filling. All the animals of this group were implanted in a single session, and 
after the first two animals, all subsequent animals developed adequate tumors.

As Group 2 was sacrificed at post-implantation day 5, 10, 15 and 20 to study tumor growth correlation at pathology and MR, this group was also used to study tumor area variations and tumor volume increase in this time frame. These variations were found to be systematic with minimal erratic behavior and a predictable growth rate (Table 2).

Histopathological characteristics of tumors grown from F98 cells implanted in the brain of Fischer rats have previously been described. ${ }^{40-43}$ The findings reported in this work are entirely congruent with these descriptions. These tumors grow by diffusely infiltrating the surrounding brain. They demonstrate extensive areas of necrosis and vascular proliferation. All these features are also characteristically found in human high-grade astrocytomas. ${ }^{44}$ On immunohistochemistry, GFAP expression in many of the tumor cells confirmed their astrocytic lineage. However, vimentin expression was even more profuse, being present in almost every tumor cells. As was previously postulated by Whittle et al, this observation probably suggests that the majority of tumor cells are neoplastic astrocytes in a dedifferentiated state, as vimentin expression is an early event in normal glial differentiation, while GFAP appears in later stages. ${ }^{29}$ The very high mitotic count translates the aggressiveness of this lineage. Albumin immunostaining was present in the extracellular space at the periphery of the tumors (Figure 6). This reflects the fact that the blood-brain barrier is locally disrupted by the growth of the tumor, as albumin normally is only minimally present in the interstitial space of the brain, due to its large molecular weight. Indeed, albumin immunohistochemistry has been reported to be a reliable marker of blood-brain barrier integrity. ${ }^{45}$

Many publications have reported the overexpression of TGFb1 in malignant gliomas, with a possible role in neoplastic tumor growth, and migration. ${ }^{46,47}$ Since the suppression of this protein is considered paramount in the research efforts of our laboratory, the characterization of its expression in this model was considered as an important objective. In this model, TGF-b1 depicted predominant regional staining around tumor blood vessels. Occasional astrocytic tumor cells depicted strong cytoplasmic staining. We have also documented that F98 cells produce TGF-b1 in active isoform by rt-PCR, ELISA and western blotting (unpublished data). TGF-b2 has been reported as an important local immunosuppressive factor produced by malignant astrocytic cells. ${ }^{48}$ However, TGF-b2 staining was insignificant in this model.

CD3 immunostaining was minimal on pathologic examination with only 2.8 staining cells per HPF. CD45 staining was insignificant, with only one occasional staining cell after multiple HPF counts. This observation confirms the weakly immunogenic properties of the F98 cells after their implantation into the brain of the Fischer rat, and the fact that the implantation procedure did not generate a significant inflammatory reaction. However, the importance of working in a syngeneic context cannot be over-emphasized. We have already demonstrated that even while using the F98 cell line, which is low immunogenic, in an allogenic setup, a significant inflammatory reaction was detected. This ultimately was associated with a tumor graft rejection, and a decrease tumor take. ${ }^{49}$ Thus, the risk that immunologic graft rejection will produce biases when evaluating therapeutic strategies with this animal model is minimal. This represents an advantage over some of the more widely used models, as Parsa et al. already demonstrated the important immunogenicity of the C6/Wistar model. ${ }^{6}$ Their report even concluded that the use of the C6/Wistar model is inadequate for the assessment of immunotherapy in the treatment of malignant gliomas.

Beside survival and pathologic data, useful information about the efficacy of different treatments can be obtained by imaging studies. Radiological evaluation allows a regular in vivo assessment of the evolution of the tumors, without sacrifice of the animal. The MRI evaluation is the gold standard in neurooncology for tumor follow-up, and should also be used in preclinical studies. Dedicated high-field MR devices generate the best quality imaging, but are expensive and not widely available. ${ }^{10,50}$ We have developed a method to image the animals at relatively low cost, using a standard clinical $1.5 \mathrm{~T}$ magnet. ${ }^{51}$ Each MRI study generated adequate resolution images both for T1 and T2-weighted imaging. Tumor boundaries were well seen on T1 images after gadolinium enhancement. The volume measurements obtained showed a gradual increase that was proportional to time from implantation, as was expected (Figure 7 and Table 2). Interestingly, when results were plotted on a logarithmic scaled graphic, tumor volumes at day 5, 10 and 15 depicted a near-perfect exponential relationship with a doubling time of 3.05 days (Figure 8). Using this function, the extrapolated initial implantation volume was estimated at 1.85 $\mathrm{mm}^{3}$. A threshold was observed after 15 days, thus implying that tumor growth gradually decreases after day 15 postimplantation. This effect is likely related to the increase interstitial pressure in and around the tumor, and the incompetence of the vascular supply past a certain volume to accommodate tumor growth.

A constant overestimation of tumor volumes compared to the pathological samples was observed. The reasons for this discrepancy are multiple: a shrinking factor produced by tissue fixation at pathology and the enhancement on MR at the brain around tumor area, related to the presence of neovascularization are the major mechanisms at play. ${ }^{52}$ The use of an image analysis software allowed a precise and quantitative characterization of tumor volumes, suggesting that it could be a useful adjunct method for the evaluation of the impact of a therapeutic strategy by allowing volumetric comparisons of the tumors between treatment groups in living animals.

Imaging with PET scan using an adequate tracer is another non-invasive technique to evaluate the tumors metabolic status in vivo. ${ }^{18} \mathrm{~F}$-FDG uptake was increased in the tumors compared to adjacent brain, conferring a way to outline the neoplastic nodule with a satisfactory degree of precision. Radiotracer uptake by the Harderian glands, exocrine glands located in the medial aspect of the orbit whose functions are not yet clearly established, was also strong. ${ }^{13}$ However, it did not obscure neoplastic uptake in such a way as to hinder adequate delineation of the tumors, as we took great care in the establishment of our implantation coordinates to implant at a significant distance from these glands. These results are consistent with the extremely malignant behavior of the model, as FDG-PET has been demonstrated to be a reliable prognostic tool for pathology and 
survival in human low and high grade astrocytomas. ${ }^{53,54}$ Tumorto-brain uptake ratio increased over time after the isotope injection, which reflects the normal clearance of the isotope from the brain, and selective sequestration in tumor cells. These results are in conflict with those obtained by Wang et al, who reported a poor contrast imaging between the brain and tumor, with blurred tumor images. ${ }^{55}$ This group reported a tumor to normal brain ratio of 1.44 for the ${ }^{18} \mathrm{~F}-\mathrm{FDG}$, while we obtained a ratio of $1.97 .{ }^{11} \mathrm{C}$-acetate is a marker of oxidative metabolism also used in myocardial perfusion studies that has shown potential use in tumor imaging. ${ }^{56}$ However, in this experiment, its uptake was not significantly different between brain and tumor, with an uptake ratio of only 1.12 . Therefore, this isotope was not considered suitable for metabolic evaluation of the F98Fischer model.

Animal models are necessary for the preclinical evaluation of antitumoral therapeutic strategies. The syngeneic Fischer/F98 rat glioma model described in this study adequately mimics the clinical and pathological behaviour of human high-grade astrocytic tumors. Careful and systematic implantation techniques, and the use of a stereotactic apparatus and microinfusion pump, contribute to produce a predictive and constant growth pattern that allows an accurate assessment of treatment. The use of the micro-infusion pump allows delivery of the implantation solution at a constant flow, thus minimizing local tissue trauma at the time of infusion. We believe that a meticulous attention to all the steps involved in the implantation is important, as the accuracy and reproducibility of the model is at stake. Ultimately, results of therapeutic experiments will be biased if the model is inadequate. Using the parameters evoked in this study, a median survival of 26 days is obtained, with a gradual, constant and predictable growth in tumors as documented pathologically and radiologically. This survival length is ideal for therapeutic trials as it allows a sufficient timewindow to treat the animals, and the effect of treatment can be recognized soon enough by using prolongation of survival as a surrogate. Moreover, magnetic resonance imaging and PET imaging studies can easily be accomplished to complement the clinical and pathological data and generate a thorough evaluation and understanding in the tumor growth of the model, whether it is for the purpose of a therapeutic trial or for detailed characterization.

\section{ACKNOWLEDGEMENT}

This work was supported by a grant from the Canadian cancer research society, and by a support grant from the Sherbrooke University Surgery department.

\section{REFERENCES}

1. Binder DK, Keles GE, Aldape K, Berger MS: Aggressive glial neoplasms. In Batjer HH, Loftus CM, editors. Textbook of neurological surgery, principles and practice, Philadelphia: Lippincott Williams \& Wilkins; 2003. p. 1270-80.

2. Barth RF. Rat brain tumor models in experimental neuro-oncology. The 9L, C6, T9, F98, RG2(D74), RT-2 and CNS-1 Gliomas. J Neurooncol. 1998;36:91-102.

3. Lampson LA. New animal models to probe brain tumor biology, therapy, and immunotherapy: advantages and remaining concerns. J Neurooncol. 2001; 53:275-87.
4. Peterson DL, Sheridan PJ, Brown Jr WE. Animal models for brain tumors: historical perspectives and future directions. J Neurosurg. 1994;80:865-76.

5. Rama B, Spoerri O, Holzgraefe M, Mennel HD.Current brain tumour models with particular consideration of the transplantation techniques. Outline of literature and personal preliminary results. Acta Neurochir. 1986;79:35-41.

6. Parsa AT, Chakrabarti I, Hurley PT, Chi JH, Hall JS, Kaiser MG, et al. Limitations of the C6/Wistar rat intracerebral glioma model: Implications for evaluating immunotherapy. Neurosurgery. 2000;47:993-1000.

7. Fournier E, Passirani C, Montero-Menei C, Colin N, Breton P, Sagodira $S$, et al. Therapeutic effectiveness of novel 5fluorouracil-loaded poly(methylidene malonate 2.1.2)-based microspheres on F98 glioma-bearing rats. Cancer. 2003; 97:2822-29.

8. Raila FA, Bowles AP, Perkins E, Terrell A. Sequential imaging and volumetric analysis of an intracerebral C6 glioma by means of a clinical MRI system. J Neurooncol. 1999;43:11-7.

9. San-Galli F, Vrignaud P, Robert J. Assessment of the experimental model of transplanted C6 glioblastoma in Wistar rats. J Neurooncol. 1989;7:299-304.

10. Thorsen F, Ersland L, Nordli H, Enger PO, Huszthy PC, Lundervold A, et al. Imaging of experimental rat gliomas using a clinical MR scanner. J Neurooncol. 2003;63:225-31.

11. Grobben B, De Deyn PP, Slegers H. Rat C6 glioma as experimental model system for the study of glioblastoma growth and invasion. Cell Tissue Res. 2002; 310:257-70.

12. Tonn JC. Model systems in neurooncology. Acta Neurochir. 2002;83:79-83.

13. Buzzell GR. The Harderian Gland: Perspectives. Microsc Res Tech. 1996; 34:2-5.

14. Lecomte R, Cadorette J, Richard P, Rodrigue S, Rouleau D. Design and engineering aspects of a high resolution positron tomograph for small animal imaging. IEEE Trans Nucl Sci. 1994;41: 1446-52.

15. Lecomte R, Cadorette J, Rodrigue S, Lapointe D, Rouleau D, Bentourkia, et al. Initial results from the Sherbrooke avalanche photodiode positron tomography. IEEE Trans Nucl Sci. 1996;43:1952-57.

16. Marriott CJ, Cadorette JE, Lecomte R, Scasnar V, Rousseau J, Van Lier JE. High resolution PET imaging and quantitation of pharmaceutical biodistributions in a small animal using avalanche photodiodes detectors. J Nucl Med. 1994; 35: 1390-6.

17. Selivanoc V, Picard Y, Cadorette J, Rodrigue S, Lecomte R. Detector response models for statistical iterative image reconstruction in high resolution PET. IEEE Trans Nucl Sci. 2000;47:1168-75.

18. Aas AT, Brun A, Blennow C, Stromblad S, Salford LG. The RG2 rat glioma model. J Neurooncol. 1995;23:175-83.

19. Auer RN, Del Maestro RF, Anderson R. A simple and reproducible experimental in vivo glioma model. Can J Neurol Sci. 1981;8:325-31.

20. Beauchesne P, Bertrand S, Revel R, Pialat J, Brunon J, Mornex F, et al. Development of an intracerebral glioma model in whole body irradiated hairless rats. Anticancer Res. 2000;20:703-6.

21. Engebraaten O, Hjortland GO, Hirschberg H, Fodstad O. Growth of precultured human glioma specimens in nude rat brain. $\mathrm{J}$ Neurosurg. 1999; 90:125-32.

22. Farrell CI, Stewart PA, Del Maestro RF. A new glioma model in rat: the C6 spheroid implantation technique permeability and vascular characterization. J Neurooncol. 1987;4:403-15.

23. Kimler BF. The $9 \mathrm{~L}$ rat brain tumor model for pre-clinical investigation of radiation-chemotherapy interactions. J Neurooncol. 1994;20:103-9.

24. Kruse CA, Molleston MC, Parks EP, Schiltz PM, KleinschmidtDeMasters BK, Hickey WF. A rat glioma model, CNS-1, with invasive characteristics similar to those of human gliomas: a comparison to 9L gliosarcoma. J Neurooncol. 1994; 22:191-200.

25. Maggio WW. Rodent glioma models. Methods in Neurosciences. 1996;30:81-96. 
26. Mella O, Bjerkvig R, Schem BC, Dahl O, Laerum OD. A cerebral glioma model for experimental therapy and in vivo invasion studies in syngeneic BD IX rats. J Neurooncol. 1990;9:93-104.

27. Michailowsky C, Niura FK, do Valle AC, Sonohara S, Meneguin TD, Tsanaclis AMC. Experimental tumors of the central nervous system: standardization of a model in rats using the 9L glioma cells [in Portuguese]. Arq Neuropsiquiatr. 2003;61:234-40.

28. Senner V, Sturm A, Hoess N, Wassmann H, Paulus W. In vivo glioma model enabling regulated gene expression. Acta Neuropathol. 2000;99:603-8.

29. Whittle IR, Macarthur DC, Malcolm GP, Li M, Washington K, Ironside JK. Can experimental models of rodent implantation glioma be improved? A study of pure and mixed glioma cell line tumours. J Neurooncol. 1998;36, 231-42.

30. Zhang X, Wu J, Gao D, Fei Z, Qu Y, Jing J. Development of a rat C6 brain tumor model. Chin Med J. 2002;115:455-57.

31. Stojiljkovic M, Piperski V, Dacevic M, Rakic L, Ruzdijic S, Kanazir S. Characterization of 9L glioma model of the Wistar rat. J Neurooncol. 2003;63:1-7.

32. Wechsler W, Szymas J, Bilzer T, Hossmann KA. Experimental transplantation gliomas in the adult cat brain. 1.Experimental model and neuropathology. Acta Neurochir. 1989;98:77-89.

33. Adam JF, Elleaume H, Joubert A, Biston MC, Charvet AM, Balosso J. Synchrotron radiation therapy of malignant brain glioma loaded with an iodinated contrast agent: First trial on rats bearing F98 gliomas. Int J Radiation Oncology Biol Phys. 2003;57:1413-26.

34. Barth RF, Yang W, Coderre JA. Rat brain tumor models to assess the efficacy of boron neutron capture therapy: a critical evaluation. J Neurooncol. 2003; 62:61-74.

35. Barth RF, Yang W, Rotaru JH, Moeschberger ML, Boesel CP, Soloway AH. Enhanced survival and cure following blood-brain barrier disruption and intracarotid injection of sodium borocaptate and boronophenylalanine. Int J Radiation Oncology Biol Phys. 2000; 47:209-18.

36. Olivi A, Ewend MG, Utsuki T, Tyler B, Domb AJ, Brat DJ, et al. Interstitial delivery of carboplatin via biodegradable polymers is effective against experimental glioma in the rat. Cancer Chemother Pharmacol. 1996;39:90-6.

37. Rhines LD, Sampath P, Dolan ME, Tyler BM, Brem H, Weingart J. O6-Benzylguanine potentiates the antitumor effect of locally delivered carmustine against an intracranial rat glioma. Cancer Res. 2000;60:6307-10.

38. Shen DHY, Marsee DK, Schaap J, Yang W, Cho JY, Hinkle G, et al. Effects of dose, intervention time, and radionuclide on sodium iodide symporter (NIS)-targeted radionuclide therapy. Gene Ther. 2004;11:161-9.

39. Von Eckardstein KL, Patt S, Zhu J, Zhang L, Cervos-Navarro J, Reszka R. Short-term neuropathological aspects of in vivo suicide gene transfer to the F98 rat glioblastoma using liposomal and viral vectors. Histol Histopathol. 2001;16:735-44.

40. Ko L, Koestner A, Wechsler W. Morphological Characterization of Nitrosourea-induced glioma cell lines and clones. Acta Neuropathol. 1980;51:23-31.

41. Reifenberger G, Bilzer T, Seitz RJ, Wechsler W. Expression of vimentin and glial fibrillary acidic protein in ethylnitrosoureainduced rat gliomas and glioma cell lines. Acta Neuropathol. $1989 ; 78: 270-82$
42. Seitz RJ, Deckert M, Wechsler W. Vascularization of syngenic intracerebral RG2 and F98 rat transplantation tumors. A histochemical and morphometric study by use of ricinus communis agglutinin I. Acta Neuropathol. 1988;76:599-605.

43. Jallo GI, Volkov A, Wong C, Carson BS Sr, Penno MB. A novel brainstem tumor model: functional and histopathological characterization. Childs Nerv Syst. 2006;22:1519-25.

44. McLendon RE, Enterline DS, Tien RD, Thorstad WL, Bruner JM. Tumors of central neuroepithelial origin. In: Bigner DD, McLendon RE, Bruner JM, editors. Russel \& Rubinstein's Pathology of tumors of the nervous system. 6th ed. London: Arnold;1998. p. 307-571.

45. Poduslo JF, Curran GL, Wengenack TM, Malester B, Duff K. Permeability of proteins at the blood-brain barrier in the normal adult mouse and double transgenic mouse model of Alzheimer's disease. Neurobiol Dis. 2001;8:555-67.

46. Jachimczak P, Hessdorfer B, Fabel-Schulte K, Wismeth C, Brysch $\mathrm{W}$, Schlingensiepen KH. Transforming growth factor-betamediated autocrine growth regulation of gliomas as detected with phosphorothioate antisense oligonucleotides. Int J Cancer. 1996;65:332-7.

47. Merzak A, McCrea S, Koocheckpour S, Pilkington GJ. Control of human glioma cell growth, migration and invasion in vitro by transforming growth factor $\beta 1$. Br J Cancer. 1994;70:199-203.

48. Maxwell M, Galanopoulos T, Neville-Golden J, Antoniades HN. Effect of the expression of transforming growth factor-beta 2 in primary human glioblastomas on immunosuppression and loss of immune surveillance. J Neurosurg. 1992;76:799-804.

49. Mathieu D, Lamarche J, Fortin D. The importance of a syngeneic glioma implantation model: comparison of the F98 cell line in Fischer and Long-Evans rats. The Journal of Applied Research. 2005;5:17-25.

50. Kish PE, Blaivas M, Strawderman M, Muraszko KM, Ross DA, Ross $\mathrm{BD}$, et al. Magnetic resonance imaging of ethylnitrosourea-induced rat gliomas: a model for experimental therapeutics of low-grade gliomas. J Neurooncol. 2001;53: 243-57.

51. Blanchard J, Mathieu D, Patenaude Y, Fortin D. MR-pathological comparison in F98-Fischer glioma model using a human gantry. Can J Neurol Sci. 2006;33:86-91.

52. Wree A, Goller HJ, Beck T. Local cerebral glucose utilization in perfusion-fixed rat brains. J Neurosci Methods. 1995;58:143-9.

53. De Witte O, Lefranc F, Levivier M, Salmon I, Brotchi J, Goldman $\mathrm{S}$. FDG-PET as a prognostic factor in high-grade astrocytoma. $\mathrm{J}$ Neurooncol. 2000;49:157-63.

54. Padma MV, Said S, Jacobs M, Hwang DR, Dunigan K, Satter M, et al. Prediction of pathology and survival by FDG PET in gliomas. J Neurooncol. 2003;64:227-37.

55. Wang HE, Wu SY, Chang CW, Liu RS, Hwang LC, Lee TW, et al. Evaluation of F-18-labeled amino acid derivatives and 18F FDG as PET probes in a brain tumor-bearing animal model. Nucl Med Biol. 2005;32(4):367-75.

56. Onson SD, Welch MJ. Investigations into tumor accumulation and peroxisome proliferators activated receptor binding by F-18 and C-11 fatty acids. Nucl Med Biol. 2002;29:211-16. 